\section{Evolution of outcome measures in schizophrenia}

TOM BURNS view of the outcome in schizophrenia, and was convinced that recovery was very rare, or even impossible, and deterioration almost inevitable.

One consequence of Kraepelin's view of the long-term outcome in schizophrenia has been the persistence in psychiatric textbooks and teaching of an excessively gloomy estimate of the outcome (van Os et al, 2006). Kraepelin's perspective, like that of many current clinical psychiatrists, was shaped by his institutional experience. He spent the bulk of his professional life working with the patients who did not recover, or those who only partly recovered, and was ignorant of those who got well and moved on. In contrast, Bleuler spent long periods in conversation with patients (including discharged and recovered patients) exploring their experiences, and took a less gloomy view of the disorder.

This supplement explores the domains of outcome measurement as they have been used in schizophrenia research. It would be misleading to suggest that the evolution of these different approaches demonstrates a single, unified development. However there is something of an evolution that can be discerned which I will attempt to outline.

\section{LONG-TERM FOLLOW-UP STUDIES}

Both of Kraepelin's main proposals about schizophrenia have been subject to extensive revision. The clear distinction between schizophrenia and bipolar disorder that he introduced has been challenged (Möller, 2003), as indeed has the very coherence of the concept of schizophrenia as a disorder (Bentall \& Beck, 2004). Similarly his gloomy appraisal of the outcome has been challenged, most convincingly by careful follow-up studies. This supplement contains a series of papers which explore the sophisticated range of outcome measures (and to a lesser extent the investigatory techniques) that have come into use to explore and compare outcomes in this most complex of disorders.

\section{Randomised controlled trials v. naturalistic studies}

Hodgson et al (2007, this supplement) review the use of longer follow-up studies to determine the outcome of schizophrenia. In the past three decades randomised controlled trials (RCTs), and particularly RCTs of antipsychotic medication, have come to dominate research in schizophrenia. Most of these are short-term (many ultra-shortterm), often only weeks, and have limited follow-up (rarely beyond a year).

The classical follow-up studies spanning years and decades (Harding et al, 1987; Ciompi, 1988) confirmed the reality of recovery in a substantial proportion of people with schizophrenia. These very long-term outcomes are essentially a description of the natural history of the disorder rather than a response to any specific intervention. Not surprisingly they have been viewed as less relevant to the practising clinician.

Hodgson et al consider the newer generation of long-term studies - usually of a year or so. Many of these are RCTs rather than naturalistic observational studies and often focus on drop-out from treatment or changes in treatment as proxies for clinical response (Lieberman et al, 2005). One reason for favouring RCTs in schizophrenia is the belief that quasi-experimental studies overestimate treatment effects. However, a series of exchanges in the New England Journal of Medicine questioned this and demonstrated that effect sizes in experimental and quasi-experimental studies were remarkably similar (Concato et al, 2000). There has been something of a resurgence of cohort studies in actively treated schizophrenia, such as the European Schizophrenia Outpatient Health Outcomes (SOHO) study (Haro et al, 2003) and the Schizophrenia Care and Assessment Programme (SCAP) study (Burns et al, 2006). These often use convenient or consecutive sampling and pragmatic, simple, clinicianrated outcome measures.

Hodgson et al point to the increasing call for more naturalistic, long-term treatment data from regulatory organisations such as the National Institute for Health and Clinical Excellence (NICE) in the UK. Despite the advantages of these studies in terms of sample size and generalisability, 
they raise important methodological questions concerning design and analysis. Hodgson et al also highlight the neglected potential of post-marketing surveillance conducted by pharmaceutical companies in illuminating long-term outcomes in the era of active management.

\section{SYMPTOM OUTCOMES}

Bleuler (1950) and Jaspers (1963) explored the form of schizophrenic experiences as the essential route to understanding the disorder. Both emphasised the importance of the structure, or form, of pathological experiences rather than their content, and this diagnostic approach was codified in 1923 by Kurt Schneider in his 'first-rank' symptoms (Schneider, 1959). Schneider was concerned to improve diagnostic reliability, in particular from individual interviews, in contrast to the extended familiarity with the patient practised by Bleuler. It is unlikely that Schneider rejected Bleuler's understanding of the basic pathology of schizophrenia, rather that he thought that hallucinations, thought disorder and delusions were more likely to be identified and recorded reliably. This 'Schneiderian' approach lends itself to modern treatment trials. It is these productive symptoms that respond rapidly to successful treatment, are more obvious and more easily quantifiable.

\section{Structured symptom scales}

Mortimer (2007, this supplement) outlines the early pre-eminence of using these positive symptoms to measure outcome in schizophrenia treatment trials. Initially, composite instruments such as the Present State Examination (PSE; Wing et al, 1974), which was designed more for diagnosis than outcome measurement, were used but these were soon superseded by rating scales specifically designed to measure symptom change. Mortimer presents the three rating scales that have been most extensively used in schizophrenia trials: the Brief Psychiatric Rating Scale (BPRS; Overall \& Gorham, 1962); the Positive and Negative Syndrome Scale (PANSS; Kay et al, 1987), which was developed from the BPRS; and the single rating of Clinical Global Impression (CGI; American Psychiatric Association, 1994). Although the primary use for each of these three involves the computation of a single sumscore, the first two have sub-scales whose analysis has helped to refine and to understand possible clinical sub-categories of schizophrenia.

Mortimer points to the primacy of symptom-rated outcomes in schizophrenia. Although no longer the only outcome measures, it is often argued (perhaps with the exception of studies of cognitivebehavioural therapy) that these other outcomes (e.g. social, vocational, well-being, satisfaction) derive from successful control of the illness and do not occur without it. Symptomatic change is proposed as the key response to treatment - whether pharmacological or other.

\section{COGNITIVE AND NEURO. BIOLOGICAL OUTCOMES}

Psychopathology was for several decades the medium for trying to understand what schizophrenia was and its underlying processes. However, classical psychopathology cannot explain all the variation in response to treatment, nor indeed the persisting social disabilities. With increased sophistication in experimental and clinical psychology, the role of cognitive functioning in the natural history of schizophrenia has become a focus of intense investigation.

\section{Cognitive function as a core feature}

Kraus \& Keefe (2007, this supplement) review current understanding of cognitive functioning in schizophrenia. They point out that problems in cognitive functioning are core to the disorder (not simply consequences of either symptoms or treatments) and they remind us that both Kraepelin and Bleuler considered cognitive decline as an inherent feature of the condition. After a long period in the shadows of Schneiderian symptomatology, cognitive dysfunction has returned centre-stage with the increasing recognition (Green, 1996) that it is responsible for much social impairment. Kraus \& Keefe overview a wide battery of available tests but there is clearly still some way to go before cognitive testing is used either in routine clinical practice or in most treatment studies. They predict that testing of cognitive function will eventually not only become a part of routine outcome assessment but will probably fundamentally reshape how we conceive the disorder.

\section{Neurobiology}

Waddington (2007, this supplement) suggests that we may soon begin to realise the potential of a wide range of neurobiological indices and measurements in schizophrenia (the various forms of imaging, neurodevelopmental indices, genomics, proteomics, metabolomics and apoptotic mechanisms). He reviews the use of these techniques to explore the pathobiology of schizophrenia and their increasing potential to chart outcome. Apart from some firstepisode psychosis samples most studies to date have been cross-sectional rather than longitudinal. Although these techniques are not yet useable in prediction at the individual patient level they may soon generate useable 'biomarkers'.

\section{PATIENT-REPORTED OUTCOMES}

Despite the future potential of cognitive testing and neurobiological measures, the diagnosis and monitoring of progress in schizophrenia takes place mainly in faceto-face interviews between patient and clinician. All the outcomes mentioned so far (and, indeed, most of those to come) are rated by the clinician or researcher. However, mental illnesses are characterised predominantly by the patient's private experiences, and clinicians rely on patients recounting to make ratings. In sharp contrast to the situation in depression and anxiety there has been a much more limited development of ratings to be completed by people with schizophrenia patients themselves. McCabe et al (2007, this supplement) review such patient-reported outcomes. They explore why there has been so little work in the field, suggesting that clincians might mistrust patients' judgements because of the severity of their disorder, and why this reluctance to use patient self-reports is being overcome. Many of the same concerns have been raised in the field of social outcomes which are reviewed by Priebe et al (2007, this supplement). Despite this, patient-reported outcomes have been shown to predict service use and the increasing 'consumer' voice in mental health.

\section{Consumerism and well-being}

Patients and their families want their appraisal of the situation to be taken more seriously. They want to say what should be judged in outcomes and to have some control over their estimation. One consequence of this is the broadening of the measures used in outcomes - satisfaction 
with services, met and unmet needs, therapeutic relationships and a greater emphasis on 'real world' outcomes such as jobs and accommodation. There has also been an increased focus on assessment of personal well-being or functioning, with the development of scales for empowerment, selfesteem, sense of coherence and recovery. These outcomes are patient- or personcentred rather than disease- or disordercentred.

\section{Use of patient-reported outcomes}

McCabe et al warn against the simultaneous use of too many such scales in schizophrenia research. They point to their high level of covariance, which may indicate a single common underlying factor (probably dependent on mood). Consequently patients who report positively on therapeutic relationship are likely also to report high satisfaction with services and empowerment, etc. However, there are differences between the scales and good evidence for validity and reliability for many.

One potential development explored here is the routine use of outcome measure and McCabe et al compare the effectiveness of such scales in feedback during the clinical interview (their own work) or delayed feedback using questionnaires. A number of exciting possibilities are opening up in this area to improve communication and understanding in the clinical interview and to measure progress and outcome.

\section{CONTEXT-DEPENDENT OUTCOME MEASURES}

Outcome measurement is used clinically mainly to judge improvement in an individual patient and in research studies to judge the efficacy of a specific treatment. The rater-recorded assessments covered so far (longitudinal outcomes, symptoms, cognitive and neurobiological measures), and to some extent the patient-reported outcomes, also serve an important function in exploring the nature of the disorder itself.

These outcomes are independent of treatment and of the local social context or the system of care. However, schizophrenia is a disorder with a profound impact on the wider society and one that absorbs a major proportion of healthcare resources. A range of outcome measures have been developed which go beyond the progress of the disease in an individual or group of individuals and measure either the impact of social and clinical responses to the disease or the wider impact of the disease on society.

\section{ADVERSE DRUG EFFECTS}

Treatments are not risk free. As Hammer \& Haddad (2007, this supplement) point out antipsychotic drugs are associated with a wide range of adverse effects which can lead to distress and impaired quality of life. The psychological price that patients may pay for a reduced risk of relapse, or for a prompt reduction in acute symptoms, can include tiredness, sluggish thinking, or even frank depression. Added to this are physical problems such as stiffness, akathisia, reduced sexual functioning and a whole range of longer-term hormonal and weight problems. These are a significant burden on individuals who already have schizophrenia to contend with. These adverse effects can also lead to poor adherence to medication if patients do not consider the benefits of the medication to outweigh the adverse effects. Some of these side-effects are also obvious to others (e.g. stiffness, tremor, weight gain) and can contribute to stigma.

Hamman \& Haddad outline the various neglected sources of data on adverse effects and the inconsistency of their recording. They argue for greater attention to these outcomes (and patients certainly do consider them outcomes) and for the need to obtain information from all possible sources rather than stick to a single protocol. Like Hodgson et al they believe that total discontinuation rates for antipsychotics (for whatever reason - poor clinical response or side-effects, or poor clinical response and side-effects) are an increasingly practical and valid outcome measure in schizophrenia management. They argue for the reporting of adverse effects in clinically, rather than statistically, meaningful ways (e.g. the number of patients who became obese during a trial rather than the mean weight gain, the number of patients who developed significant akathisia rather than the mean increase in akathisia score). They propose that more careful and routine measurement of side-effects should lead to greater openness in discussing their likelihood with patients.

\section{WIDER SOCIETAL OUTCOMES}

Adverse drug effects, although dependent on local treatment regimes, are outcomes that are of direct relevance to the treated patient. There is, however, a range of outcomes that might be of equal or greater interest to others (the healthcare system, society at large) than to the patient. Kooyman et al (2007, this supplement) consider outcomes of public concern in schizophrenia. The care of people with schizophrenia is profoundly affected by infrequent but high-profile consequences of their illness. It is these dramatic and public outcomes such as violence or suicide, or socially unacceptable outcomes such as homelessness and vagrancy, that set the policy agenda and attract or deflect investment in mental healthcare.

Kooyman et al outline the major areas violence, victimisation, suicide and selfharm, substance misuse, homelessness and unemployment. For each they present what is known about the major risk factors and the methodological problems presented in attempting to measure these outcomes. Unlike patient-centred outcome measures these are mainly direct measures which do not need extensive testing of validity, sensitivity and reliability as do scales developed from psychological theory. However, the reliability and comparability of different ways of collecting these data is an equally difficult challenge - for example there is no consensus on the timescales of recording (e.g. past month, or week, or lifetime) across different statistics.

Employment or homelessness can be sensitive indicators of the adequacy of local services, and episodes of violence or suicide (albeit individually rare) can alert observers to failing services. Even if it can be argued that many of these lie outside the power of psychiatry to influence, failing to take account of them will certainly lead to criticisms of services and demands for changes.

\section{HOSPITALISATION}

Hospitalisation is one of the most common outcome measures used in mental health services research, particularly in RCTs (Catty et al, 2002). The strength of this outcome, like many of the wider societal outcomes, is that is a 'hard' outcome. Although there may be some difficulties in collecting the data consistently, it is clear what it means and it does not require much interpretation. Later in this supplement (Burns, 2007) I explore the differing ways in which hospitalisation has been used in mental health services research for 
psychoses - from simply 'admitted/not admitted', through number of admissions in a set follow-up period, to days in hospital and survival curves. In high-income countries, hospitalisation is a fairly good proxy for relapse in schizophrenia (although as Isaac et al (2007, this supplement) point out this is not so in low- and middle-income countries). The threshold will differ in different countries and healthcare systems and will depend on the quality of community care, but within a single study the difference will give a good indication of treatment effectiveness.

The problem with hospitalisation is, of course, its extreme context specificity, and I explore the risks inherent in generalising across different healthcare systems. Hospitalisation also lacks acceptability to an increasingly sceptical and critical consumer movement, as it appears to be an outcome relevant only to the service provider.

\section{DURATION OF UNTREATED PSYCHOSIS}

Early intervention in psychoses is a major concern worldwide (Edwards et al, 2000). Services are being widely established to promote earlier detection and treatment of schizophrenia and other psychoses. The rationale is not just humanitarian (the reduction in the duration of untreated distress) but a growing recognition that the duration of untreated psychosis may have a major impact both on immediate recovery rates (Marshall et al, 2005) and on longterm outcomes and disability (Larsen $e t a l$, 2006). Singh (2007, this supplement) reviews this literature and explores whether or not the duration of untreated psychosis can be sensibly used as an outcome measure in its own right.

An independent effect of the duration of untreated psychosis on outcome has been attributed to a direct 'neurotoxic' effect (Larsen et al, 2006) and to a 'critical period' in personal development when people may miss out on vital social development and may acquiring disabilities and patterns of behaviour with long-term consequences (Birchwood et al, 1998). Early intervention teams aim to reduce the duration of untreated psychosis and many have established extensive programmes of public education and outreach to achieve this.

Singh outlines the methodological problems in identifying when psychoses begin and when prodromal phases end.
He questions the representativeness of the long durations of untreated psychosis reported in earlier studies and some of the very extensive reductions reported. However, the association between duration without treatment and outcome does give some support for its use as a service-level outcome measure in schizophrenia. A less clear picture of this association has been reported from India (see Isaac et al).

\section{ECONOMIC OUTCOMES}

Early intervention services may alter helpseeking patterns and reduce subsequent reliance on in-patient care. If this proves to be the case, then early intervention promises a substantial saving in healthcare costs. McCrone's paper (2007, this supplement) on economic outcome measures in schizophrenia highlights how in-patient care accounts for a disproportionate amount of healthcare costs over the long term. Although most schizophrenia care is in the community, with brief in-patient care for acute relapses, in-patient care still accounts for most of the costs in high-income countries (although not necessarily in low- and middle-income countries - see Isaac et al). Indeed, the prominence of economic analyses in mental healthcare arose in part from the recognition that major savings could be achieved by modest shifts in the use of in-patient stays (Weisbrod et al, 1980).

McCrone outlines the range of economic outcome analyses that can be used to link costs with outcomes in schizophrenia care (cost minimisation, cost-effectiveness, cost consequence, cost-utility and cost-benefit analyses). The cost-effectiveness/cost-utility plane illustrates how judgements can be made about whether new interventions are economically indicated. However, where a more expensive approach produces a better result further consideration is needed.

Cost-utility analyses are developed to address this question using a standard outcome measure and calculating the cost of achieving one unit improvement with the treatments studied. The EuroQoL (Williams, 1995) is probably the most widely used such unit of measurement but there are few schizophrenia outcome studies using it. Quality-adjusted life-years (QALYs) have the advantage that they can be used to compare cost-utility across different areas of health and are increasingly sought by health regulatory bodies such as NICE. However, McCrone points out their lack of sensitivity in schizophrenia and current work on incremental cost-effectiveness ratios which compare the cost of achieving an agreed improvement in a chosen outcome measure (e.g. the BPRS in schizophrenia). There is still some way to go with this approach, but work is ongoing on defining 'clinically meaningful minimal changes' in terms of symptom scores, and these should help to consolidate the use of incremental cost-effectiveness ratios in economic analyses.

Economic studies of schizophrenia care highlight to politicians and policy makers just how costly the disorder is to society. Schizophrenia is unlikely to compete successfully for public attention against cancer or cardiovascular disorders, but economic evaluations demonstrate clearly that it needs to be a top priority for service improvement and treatment research.

\section{INTERNATIONAL OUTCOMES}

The evolution of outcome measures outlined in this introduction and in this supplement appears to follow a coherent and logical pattern. However, this progression - from natural history, via symptoms to more scientific ratings as the technology becomes available, alongside more subjective measures and measures of quality of life as treatments improve, ending with specific measures of schizophrenia in consistent and highly evolved services - is only one path. Isaac et al (2007, this supplement) point out that the evolution of outcome measures in low- and middle-income countries has taken a different path, and not just because of a lack of resources. The pattern of the disorder is clearly influenced by the social context (Leff et al, 1992), with differences in the severity of outcome but also differences in local priorities and values. Social functioning is less affected in low- and middle-income countries but family support and burden are much more important. Unlike the West, the economics of care are markedly different with no suggestion that outcomes such as hospitalisation have any value in comparing interventions.

Isaac et al highlight the need to adapt Western instruments for use in very different settings but also to develop local instruments. The balance is between developing instruments that are highly sensitive to local conditions with high face validity and the need to compare outcomes internationally. Their review also brings us back 
to some of the vital outcomes currently overlooked in the West - the impact on marriage and the very high early death rate in schizophrenia.

\section{CONCLUSIONS}

This supplement focuses on the varied aspects of outcome measures in schizophrenia. It has been suggested that the outcomes which are recorded and underpin decision-making have shown a clear evolution. However, this 'march of progress' view should not be taken too seriously - it is simply one way of organising the wealth of research in the area. Undoubtedly the introduction of active treatments has shifted the focus from naturalistic longterm outcome studies and improved the measurement of current status. However, the more recent developments reflect more complex drivers.

There are the enormous strides in technologies of both treatment and investigation. Effective drugs and service developments such as community mental health teams and assertive community treatment, mean that the outcome differences that must be measured are more sensitive but also broader. Most of the 'softer' outcome measures such as quality of life, social functioning and personal well-being are only of relevance in situations were symptom control is relatively well achieved. Cognitive functioning and the neurobiological parameters of the disorder have only recently come into their own as the science of their measurement matures.

As systems of care become more consistent and predictable they themselves affect the outcomes to be measured. The ubiquity of maintenance antipsychotic treatment necessitates greater attention to adverse effects of drug treament. Assessment of met needs and patient satisfaction have become important patient-centred measures of how services are functioning. The costs of these comprehensive services and the degree to which they reduce the wider social consequences associated with schizophrenia become important quality measures. With consistency of approach hospitalisation can be used as a decent proxy outcome measure for local variations in services.

The emergence of patient-reported outcomes reflects not only this increase in interest in softer outcomes, but perhaps a philosophical shift with a greater recognition of the importance of the patient's view.

TOM BURNS, MD, Social Psychiatry, University Department of Psychiatry, Warneford Hospital, Headington, Oxford OX3 7JX, UK. Email: tom.burns@psych.ox.ac.uk

It is not just that patient-reported outcomes may give a more sensitive measure of the progress of the disorder, but that people with schizophrenia are treated as agents of their management. They not only can report on how they are doing but can influence what should be the measure of how they are doing - hence the appearance of self-empowerment and general wellbeing measures in the schizophrenia literature. Nor is this restricted to subjective measures - this partnership approach has resulted in greater prominence for nonclinical hard outcomes such as employment or adequacy of accommodation, etc.

From the opposite direction (from society and healthcare funders) there is increased pressure for closer scrutiny of the broader impact of schizophrenia. The rapid growth in mental health economic outcome studies indicates increased sensitivity to the social burden of the disorder, as does the current attention paid to risk and the wider societal outcomes of violence, victimisation, suicide, etc.

However, this neat attempt at imposing order is just that - an attempt, as Isaac et al remind us. The outcomes we need to measure are not fixed. They will continue to change as society's preoccupations change, as our measurement technologies change and as treatments improve. What is clear, however, is that keeping abreast of developments in schizophrenia outcomes is a challenge for clinicians and researchers alike.

\section{REFERENCES}

American Psychiatric Association (1994) Diagnostic and Statistical Manual of Mental Disorders (4th edn) (DSM-IV). APA.

Bentall, R. \& Beck, A. (2004) Madness Explained: Psychosis and Human Nature. Penguin Books.

Birchwood, M., Todd, P. \& Jackson, C. (1998) Early intervention in psychosis. The critical period hypothesis. British Journal of Psychiatry, 172 (suppl. 33), 53-59.

Bleuler, E. (1950) Dementia Praecox or the Group of Schizophrenias (transl. J. Zinkin). International Universities Press.

Burns, T., Christova, L., Cooper, S., et al (2006) Maintenance antipsychotic medication patterns in outpatient schizophrenia patients: a naturalistic cohort study. Acta Psychiatrica Scandinavica, I13, 126-134.

Burns, T. (2007) Hospitalisation as an outcome measure in schizophrenia. British Journal of Psychiatry, I9I (suppl. 50), s37-s4l.
Catty, J., Burns, T., Knapp, M., et al (2002) Home treatment for mental health problems: a systematic review. Psychological Medicine, 32, 383-40।.

Ciompi, L. (1988) Learning from outcome studies. Toward a comprehensive biological-psychosocial understanding of schizophrenia. Schizophrenia Research, I, 373-384.

Concato, J., Shah, N. \& Horwitz, R. I. (2000) Randomized, controlled trials, observational studies and the hierarchy of research designs. New England Journal of Medicine, 342, 1887-1892.

Edwards, J., McGorry, P. D. \& Pennell, K. (2000)

Models of early intervention in psychosis: an analysis of service approaches. In Early Intervention in Psychosis: A Guide to Concepts, Evidence and Interventions (eds M. Birchwood, D. Fowler \& C. Jackson), pp. 28I-3|4. John Wiley \& Sons.

Green, M. F. (1996) What are the functional consequences of neurocognitive deficits in schizophrenia? American Journal of Psychiatry, 153 $321-330$.

Hamer, S. \& Hadad, P. M. (2007) Adverse effects of antipsychotics as outcome measures. British Journal of Psychiatry, 19I (suppl. 50), s64-s70.

Harding, C. M., Brooks, G.W., Ashikaga, T., et al (1987) The Vermont longitudinal study of persons with severe mental illness. Il. Long-term outcome of subjects who retrospectively met DSM-III criteria for schizophrenia. American Journal of Psychiatry, 144, 727 735 .

Haro, J. M., Edgell, E. T., Jones, P. B., et al (2003) The European Schizophrenia Outpatient Health Outcomes $(\mathrm{SOHO})$ study: rationale, methods and recruitment. Acta Psychiatrica Scandinavica, 107, 222-232.

Hodgson, R., Bushe, C. \& Hunter, R. (2007) Measurement of long-term outcomes in observational and randomised controlled trials. British Journal of Psychiatry, 191 (suppl. 50), s78-s84.

Isaac, M., Chand, P. \& Murthy, P. (2007) Schizophrenia outcome measures in the wider international community. British Journal of Psychiatry, 19I (suppl. 50), s7I-s77.

Jaspers, K. (1963) General Psychopathology (transl. J. Hoenig \& M.W. Hamilton). Manchester University Press.

Kay, S. R., Fiszbein, A. \& Opler, L. A. (1987) The positive and negative syndrome scale (PANSS) for schizophrenia. Schizophrenia Bulletin, I3, 26I-276.

Kooyman, I., Dean, K., Harvey, S., et al (2007) Outcomes of public concern in schizophrenia. British Journal of Psychiatry, 19I (suppl. 50), s29-s36.

Kraepelin, E. (1919) Dementia Praecox and Paraphrenia (transl. R. M. Barclay) (197I edn). Kreiger.

Kraus, M. S. \& Keefe, R. S. E. (2007) Cognition as an outcome measure in schizophrenia. British journal of Psychiatry, 19I (suppl. 50), s46-s5l.

Larsen, T. K., Melle, I., Auestad, B., et al (2006) Early detection of first-episode psychosis: the effect on I-year outcome. Schizophrenia Bulletin, 32, 758-764.

Leff, J., Sartorius, N., Jablensky, A., et al (1992) The International Pilot Study of Schizophrenia: five-year follow-up findings. Psychological Medicine, 22, |3|-|45. 
Lieberman, J. A., Stroup, T. S., McEvoy, J. P., et al (2005) Effectiveness of antipsychotic drugs in patients with chronic schizophrenia. New England Journal of Medicine, 353, 1209-1223.

Marshall, M., Lewis, S., Lockwood, A., et al (2005) Association between duration of untreated psychosis and outcome in cohorts of first-episode patients: a systematic review. Archives of General Psychiatry, 62. 975-983.

McCabe, R., Saidi, M. \& Priebe, S. (2007) Patientreported outcomes in schizophrenia. British journal of Psychiatry, 191 (suppl. 50), s2l-s28.

McCrone, P. (2007) Health economic measures in schizophrenia research. British Journal of Psychiatry, 191 (suppl. 50), s42-s45.

Möller, H.-J. (2003) Bipolar disorder and schizophrenia: distinct illnesses or a continuum? Journal of Clinical Psychiatry, 64 (suppl. 6), 23-27.
Mortimer, A. M. (2007) Symptom rating scales and outcome in schizophrenia. British Journal of Psychiatry, I9I (suppl. 50), s7-sl4.

Overall, J. E. \& Gorham, D. L. (1962) The Brief Psychiatric Rating Scale. Psychological Reports, 10, 799 812

Priebe, S. (2007) Social outcomes in schizophrenia British Journal of Psychiatry, 19I (suppl. 50), sl5-s20.

Schneider, K. (1959) Clinical Psychopathology (transl. M.W. Hamilton). Grune \& Stratton.

Singh, S. P. (2007) Outcome measures in early psychosis. Relevance of duration of untreated psychosis. British Journal of Psychiatry, 19I (suppl. 50), s58-s63.

van Os, J., Burns, T., Cavallaro, R., et al (2006) Standardized remission criteria in schizophrenia. Acta Psychiatrica Scandinavica, II3, 91-95.
Waddington, J. L. (2007) Neuroimaging and other neurobiological indices in schizophrenia: relationship to measurement of functional outcome. British journal of Psychiatry, 191 (suppl. 50), s52-s57.

Weisbrod, B. A., Test, M. A. \& Stein, L. I. (1980) Alternative to mental hospital treatment. II. Economic benefit-cost analysis. Archives of General Psychiatry, 37, 400-405.

Williams, A. (1995) The Role of the EuroQoL Instrument in QALY Calculations. Centre for Health Economics, University of York.

Wing, J. K., Cooper, J. E. \& Sartorius, N. (1974) The Measurement and Classification of Psychiatric Symptoms. An Instruction Manual for the PSE and the Catego

Program. Cambridge University Press. 